\title{
EFFECT OF A SPECULUM BLADE ON DYNAMIC MRI EVALUATION OF PELVIC ORGAN PROLAPSE
}

Hypothesis / aims of study

To evaluate whether the use of a speculum blade modifies the evaluation of pelvic organ prolapse as assessed by dynamic MRI.

Study design, materials and methods

Women with POP-Q stage II or greater, who were due to undergo genital prolapse surgery, were evaluated using dynamic MRI. The procedure was repeated using the posterior blade of a standard plastic Grave's speculum to successively retract the anterior and posterior vaginal walls. Pelvic organ position, prolapsed compartments, and prolapse degree were evaluated according to the use of a speculum blade

\section{$\underline{\text { Results }}$}

Twenty seven patients were enrolled in this pilot study. Standard POP-Q was $15 \%$ stage II, $59 \%$ stage III, and $26 \%$ stage IV prolapse.

The use of a blade evidenced hidden pelvic prolapsed compartments in $59 \%(n=16)$ of cases. For $48 \%$ of patients ( $n=13)$, the variation of the leading edge of at least one additional prolapsed compartment was diagnosed as more than $20 \mathrm{~mm}$. Using a speculum blade, patients were significantly more often diagnosed with POP of more than one compartment $(p=0.0015)$.

Interpretation of results

The use of a speculum blade during dynamic MRI modifies the pelvic organ prolapse evaluation in a large proportion of patients who require prolapse surgery.

\section{Concluding message}

The results of this study point to a role for the use of a speculum blade during MRI POP evaluation.

(2 references)

References

1. Delaney S, Fernandez P, N'Guyen A, Salomon L, Carbonne B, Laissy JP, Ansquer Y. Effetc of a speculum blade on dynamic MRI evaluation of pelvic organ prolapse. Acta Obstet Gynaecol Scand 2009; In Press

2. Comiter C, Vasavada S, Barbaric Z, Gousse A, Raz S. Grading pelvic prolapse and pelvic floor relaxaxation using dynamic magnetic resonance imaging. urology 1999; 54: 454-7

\begin{tabular}{ll}
\hline Specify source of funding or grant & no souce of funding or grant \\
\hline Is this a clinical trial? & No \\
\hline What were the subjects in the study? & HUMAN \\
\hline Was this study approved by an ethics committee? & Yes \\
\hline Specify Name of Ethics Committee & Comité d'Ethique de la recherche en Gynécologie Obstétrique \\
\hline Was the Declaration of Helsinki followed? & Yes \\
\hline Was informed consent obtained from the patients? & Yes \\
\hline
\end{tabular}

